\title{
A simple finite-time distributed observer design for linear time-invariant systems ${ }^{\star}$
}

\author{
Haik Silm ${ }^{\mathrm{a}, \mathrm{c}, *}$, Denis Efimov ${ }^{\mathrm{b}, \mathrm{d}}$, Wim Michiels ${ }^{\mathrm{c}}$, Rosane Ushirobira ${ }^{\mathrm{b}}$, Jean-Pierre Richard $^{\mathrm{a}}$ \\ ${ }^{a}$ Univ. Lille, CNRS, Inria, Centrale Lille, UMR 9189 - CRIStAL, 59000 Lille, France \\ ${ }^{b}$ Inria, Univ. Lille, CNRS, UMR 9189 - CRIStAL, 59000 Lille, France \\ ${ }^{c}$ Department of Computer Science, KU Leuven, 3001 Heverlee, Belgium \\ ${ }^{d}$ ITMO University, 197101 Saint Petersburg, Russia
}

\begin{abstract}
A design of a distributed observer is proposed for continuous-time systems with nonlinear observer nodes such that the estimation errors converge in a finite time to zero. By taking advantage of individual observability decompositions, the designs for the locally observable and the unobservable substate are made independent from each other. For the observable substate of each node, standard centralized finite-time observer techniques are applied. To estimate distributively the unobservable substate, the observer nodes employ consensus coupling in a linear term and an additional term embedded in a fractional power. The approach is derived using homogeneity arguments and it leads to a simple design with an LMI that is guaranteed to be feasible under general conditions.
\end{abstract}

Keywords: Distributed estimation, Linear systems, Finite-time observers, Homogeneity

\section{Introduction}

The distributed observer design problem is concerned with reconstructing the state of a linear time-invariant system from its output using a network of state observer nodes. The goal is to have a full state estimate at each node of the network. The constraint is that not every node has direct access to the complete output of the system, but instead the output vector is divided between the set of nodes. The division is such that it might be impossible for a single node to reconstruct the state of the system using a standard centralized observer, since the system is not observable from a partial output. This requires that an observer node is connected to other nodes and collaborates with them to obtain the missing information. The observer nodes do not directly communicate with every other node but only with a subset of given neighboring nodes. This is accounted for by an apriori given communication graph.

The imposed constraint, that is the lack of local observability (contrary to the common case in sensor fusion) under limited communication (making centralized approaches unfeasible), is challenging. Recently in [1] the distributed observer design problem was solved for discrete time linear systems under nonrestrictive conditions. By

${ }^{\star}$ This work has been partially supported by the project UCoCoS, funded by the European Union's Horizon 2020 research and innovation program under the Marie Skłodowska-Curie Grant Agreement No 675080. It was also partially funded by Russian Science Foundation (Project 17-19-01422).

* Corresponding author

Email address: haik.silm@kuleuven.be (Haik Silm) nonrestrictive, we mean assumptions on the local output matrices and the communication graph that are necessary for any kind of distributed observer design. Next to establishing these conditions is the question of how to design the observer nodes with respect to a certain performance criterion. The goal of this paper is to design a distributed observer for continuous-time systems, such that the estimates of the observer nodes reach the state in a finite time.

To this end, we propose a design based on homogeneity [2], which can be contrasted to linear designs that can attain only asymptotic convergence of the estimation error. Homogeneity based centralized finite-time observer design relies on the fact that the dynamics of the error system can be sort of eliminated with a preliminary linear feedback, resulting into a nilpotent matrix (which for a single output system can be chosen to be an integrator chain). However, this requires that the system is observable from the output, which is exactly not fulfilled in the distributed observer setting. Therefore, in a previous note on designing finitetime observer in the distributed framework, we restricted ourselves to a limited class of systems [3]. The new design is feasible for general linear systems.

Since this work relies on earlier results on consensusbased distributed observer design, a short summary of the milestones of this approach is given. First, in [4] the idea of taking the standard Luenberger observer design and complement it with a consensus term was presented. However, for some time it has not been clear under which conditions this approach works and how to choose the gains. Some LMI based results for the design have been presented [5], however there was no indication of their feasibility. An 
important contribution was made in 6 where it was proposed to apply the observability decomposition on each node, to thus refine the selection of the consensus matrices. The fundamental idea is that for each node only the component which is not in its unobservable subspace acts on the corresponding part of the output. Therefore, if the output feedback term is restricted to the observable subspace, it is possible to select the Luenberger observer gain such that the eigenvalues of the error system for this part are stable. For the additional consensus term, a sufficiently high scalar consensus gain has to be selected only for the unobservable subspaces. The same structure was chosen in 7 and using the notion of balanced mirror graph it was shown that this design works under the condition of joint observability and strong connectedness of the directed communication graph.

A more refined selection of consensus gains matrices was derived in [8] by applying a multi-hop subspace decomposition, where in turn the conditions on the neighborhood relations were extended to general directed graphs. This formulation allows to assign the poles of error system of the observer network. As such it is also applicable to discretetime systems. However, it is on the expense of a more complex design compared to the observability decomposition with a scalar gain used in this work. There are other methods which allow selecting the poles in the discrete time-setting and therefore to accomplish finite-time convergence [9] (akin to a dead-beat observer in the centralized setting). Pole placement for continuous-time systems does also open up the possibility to design finite-time distributed observers using generalized homogeneity concepts [10, nonetheless the approach of the present paper using just standard observability decompositions offers a design which is comparatively simple.

The structure of this paper is as follows: In Section 2 we first formally describe the distributed observer design problem for a linear system. Next, we present a known linear distributed observer design using consensus and observability decompositions for asymptotic convergence and show that it solves the problem for a jointly observable system with a strongly connected communication graph. To this end, we establish the error system for the observer network, prove that it is asymptotically stable and moreover that the Lyapunov matrix can be selected to be diagonal. Building upon this, we present in Section 3 the finite-time distributed observer design for the observable and the unobservable subspaces and justify them using homogeneity arguments. For the main result of distributed finite-time observer for the unobservable subspaces, we propose a Lyapunov function which relies on the diagonal stability property established before. Finally, we give an illustrative example and concluding remarks in Section 4 and Section 5 . respectively.

\section{Notation}

- For $a=\left[\begin{array}{lll}a_{1} & \cdots & a_{n}\end{array}\right]^{\top} \in \mathbb{R}^{n}$ and $b=$ $\left[\begin{array}{lll}b_{1} & \cdots & b_{n}\end{array}\right]^{\top} \in \mathbb{R}_{+}^{n}$ we denote by $\lceil a\rfloor^{b}=$ $\left[\operatorname{sgn}\left(a_{1}\right)\left|a_{1}\right|^{b_{1}} \quad \cdots \quad \operatorname{sgn}\left(a_{n}\right)\left|a_{n}\right|^{b_{n}}\right]^{\top}$ the sign preserving element-wise exponentiation.

- $\operatorname{diag}(x)$ with $x=\left[\begin{array}{lll}x_{1} & \cdots & x_{n}\end{array}\right]^{\top} \in \mathbb{R}^{n}$ denotes the diagonal matrix with $x_{1}, \ldots, x_{n}$ on the main diagonal.

- $\operatorname{blkdiag}\left(A^{1}, \ldots, A^{N}\right)$ denotes a block-diagonal matrix with matrices $A^{1}, \ldots, A^{N}$ as diagonal blocks.

- $\mathbf{1} \in \mathbb{R}^{n}$ is the vector with all entries equal 1 and $I$ is the identity matrix of appropriate dimension.

- $\otimes$ denotes the Kronecker product.

- $\mathcal{L}_{\infty}$ is the set of essentially bounded measurable functions $\mathbb{R} \rightarrow \mathbb{R}^{m}$ with the norm $\|\cdot\|_{[0,+\infty)}=$ $\sup _{t \in[0,+\infty)}\|\cdot\|$, where $\|\cdot\|$ denotes the Euclidean norm on $\mathbb{R}^{n}$.

\section{Consensus-based distributed observer design}

We consider linear systems with multiple outputs of the form

$\dot{x}=A x, \quad y^{1}=C^{1} x, \ldots, y^{N}=C^{N} x$,

where $x \in \mathbb{R}^{n}$ is the state to be estimated, and a distributed observer consisting of a set of $N$ observer nodes, each corresponding to one of the possible vector-valued outputs $y^{i} \in \mathbb{R}^{m_{i}}, i \in\{1, \ldots, N\}$. The only assumption we make on the system is that the output matrices $C^{i}$ are such that its state can be reconstructed from the outputs if they are all measured together:

Assumption 1. The system (1) is jointly observable (i.e. the observability matrix $\mathcal{O}\left(A,\left[C^{1^{\top}} \cdots C^{N^{\top}}\right]^{\top}\right)$ has full rank).

The aim of a distributed observer is to reconstruct the full state of the plant at each observer node, under the constraint that this is not possible using a single output (i.e. for a linear system the pair $\left(A, C^{i}\right)$ is neither observable nor detectable). To be able to do so, the observer nodes have to collaborate with each other by communicating over a network. We describe this network by a simple unweighted directed graph $\mathcal{G}=(\mathcal{V}, \mathcal{E})$ of order $|\mathcal{V}|=N$, where $\mathcal{V}$ and $\mathcal{E}$ denote the set vertices and edges of $\mathcal{G}$, respectively. Each vertex holds an observer node and is labeled by an index in $\mathcal{V}=\{1, \ldots, N\}$ corresponding to the output of the system which it measures. The set of neighbors $\mathcal{N}_{i}=\{j:(j, i) \in \mathcal{E}\}$ of a vertex $i$ is constituted by other observer nodes it receives additional information from. The graph is also fully characterized by its Laplacian matrix $\mathcal{L}=\mathcal{A}-\mathcal{D}$, where for a directed graph the adjacency matrix $\mathcal{A}=\left[a_{i j}\right]$ is defined with $a_{i j}=1$ if there is an outgoing edge from $j$ to $i$ (otherwise zero), and $\mathcal{D}=\operatorname{diag}\left(\left|\mathcal{V}_{1}\right|, \ldots,\left|\mathcal{V}_{N}\right|\right)$ is the in-degree matrix. 


\subsection{Observability decomposition}

A straightforward solution to the distributed observer design problem would be to distribute the individual measurements of the nodes over the whole observer network and implement each node as a centralized observer. This would be a viable solution if there are many direct connections between the nodes, such that each node together with its neighbors is jointly observable. Otherwise each node forwards not only its own measurement, but also the measurement it receives from its neighbors. In such a case the measurements which have to go over multiple nodes might be impacted by large delays, deteriorating the estimation performance [11]. Therefore, an alternative to distributing the measurements is that each observer node exchanges its state estimate with its neighbors. The problem of limited connectivity is then overcome by using consensus feedback as will be shown in this section. One drawback is that for a large-scale system the full state estimate can be of a high dimension. However, this can be alleviated by the fact that each node has already some information of the state of the system by its measurement input, therefore it does not have to receive the full state estimate from its neighbors but only for the part which it cannot reconstruct on its own.

This motivates to apply the well-known observability decompositions at each node using orthogonal state transformations $x^{i}=T^{i}\left[\begin{array}{c}x_{o}^{i} \\ x_{u}^{i}\end{array}\right], T^{i}=\left[\begin{array}{ll}T_{o}^{i} & T_{u}^{i}\end{array}\right]$, where the columns of $T_{u}^{i}$ form a basis of the kernel of the observability matrix of the pair $\left(A, C^{i}\right)$. This leads with

$T^{i^{\top}} A T^{i}=\left[\begin{array}{cc}A_{o}^{i} & 0 \\ A_{r}^{i} & A_{u}^{i}\end{array}\right], \quad C^{i} T^{i}=\left[\begin{array}{ll}C_{o}^{i} & 0\end{array}\right]$

to a decomposition of the system into an observable subsystem, which by construction is observable from the corresponding output (the pair $\left(A_{o}^{i}, C_{o}^{i}\right)$ is observable), and a coupled unobservable subsystem [12. Consequently, for the observable part an observer node can rely on its measurement input, but to reconstruct its unobservable part it has to obtain additional information form the other nodes. To this end, the output-injection term of a Luenberger observer is complemented with diffuse coupling over the neighbourhood $\mathcal{N}_{i}$ by exchanging their state estimates as follows

$$
\dot{\tilde{x}}^{i}=A \tilde{x}^{i}-T_{o}^{i} L_{o}^{i}\left(C_{i} \tilde{x}^{i}-y^{i}\right)-\alpha_{i} T_{u}^{i} T_{u}^{i^{\top}} \sum_{j \in \mathcal{N}_{i}}\left(\tilde{x}^{i}-\tilde{x}^{j}\right)
$$

where $\tilde{x}^{i} \in \mathbb{R}^{n}$ is the estimate of $x$ generated by the observer node which has access to the output $y^{i}$, and the Luenberger observer gain $L_{o}^{i}$ and consensus gain $\alpha_{i} \in \mathbb{R}$ appear as design parameters. Note that if a node $j$ knows the value of $T_{u}^{i}$ of its neighbor $i$, it can just transmit the projection $T_{u}^{i^{\top}} \tilde{x}^{j}$ instead of $\tilde{x}^{j}$ to reduce the size of the vector.

Together with (2), the observer algorithm (3) can be expressed in local coordinates of the estimate $\tilde{x}_{o}^{2}$ and $\tilde{x}_{u}^{i}$ for the observable

$\dot{\tilde{x}}_{o}^{i}=A_{o}^{i} \tilde{x}_{o}^{i}-L_{o}^{i}\left(C_{o}^{i} \tilde{x}_{o}^{i}-y^{i}\right)$

and unobservable part

$\dot{\tilde{x}}_{u}^{i}=A_{r}^{i} \tilde{x}_{o}^{i}+A_{u}^{i} \tilde{x}_{u}^{i}-\alpha_{i} \sum_{j \in \mathcal{N}_{i}}\left(\tilde{x}_{u}^{i}-T_{u}^{i^{\top}} \tilde{x}^{j}\right)$,

respectively, where $\tilde{x}^{i}=T_{o}^{i} \tilde{x}_{o}^{i}+T_{u}^{i} \tilde{x}_{u}^{i}$. In the next subsection we will analyze the resulting error system to show that it leads to an asymptotically converging observer if the communication graph is strongly connected (there exist a sequence of outgoing edges from each vertex to every other vertex). This will be the basis for the modifications in the next section to obtain a finite-time distributed observer.

\subsection{Error system analysis}

To show under which conditions the local observers (4) and (5) converge to the correct state, we have to investigate the behaviour of the estimation error of the observer network. We do this in local coordinates by defining $e_{o}^{i}=\tilde{x}_{o}^{i}-x_{o}^{i}$ and $e_{u}^{i}=\tilde{x}_{u}^{i}-x_{u}^{i}$, to obtain the error system at each node as

$\dot{e}_{o}^{i}=\left(A_{o}^{i}-L_{o}^{i} C_{o}^{i}\right) e_{o}^{i}$,

$\dot{e}_{u}^{i}=A_{r}^{i} e_{o}^{i}+A_{u}^{i} e_{u}^{i}-\alpha_{i} \sum_{j \in \mathcal{N}_{i}}\left(e_{u}^{i}-T_{u}^{i^{\top}}\left(T_{o}^{j} e_{o}^{j}+T_{u}^{j} e_{u}^{j}\right)\right)$,

for the observable and unobservable subspace, respectively. Concatenating them in a rearranged order $E_{o}^{\top}=$ $\left[\begin{array}{ccc}e_{o}^{i^{\top}} & \cdots & e_{o}^{N^{\top}}\end{array}\right], E_{u}^{\top}=\left[\begin{array}{lll}e_{u}^{1^{\top}} & \cdots & e_{u}^{N^{\top}}\end{array}\right]$, we obtain the error system for the whole observer network in a block triangular form

$$
\left[\begin{array}{c}
\dot{E}_{o} \\
\dot{E}_{u}
\end{array}\right]=\left[\begin{array}{cc}
\boldsymbol{A}_{\boldsymbol{o}}-\boldsymbol{L}_{\boldsymbol{o}} \boldsymbol{C}_{\boldsymbol{o}} & 0 \\
\boldsymbol{A}_{\boldsymbol{r}}+\boldsymbol{\alpha} \boldsymbol{T}_{\boldsymbol{u}}^{\boldsymbol{\top}}(\mathcal{A} \otimes I) \boldsymbol{T}_{\boldsymbol{o}} & \boldsymbol{A}_{\boldsymbol{u}}-\boldsymbol{\alpha} \boldsymbol{T}_{\boldsymbol{u}}^{\boldsymbol{\top}}(\mathcal{L} \otimes I) \boldsymbol{T}_{\boldsymbol{u}}
\end{array}\right]\left[\begin{array}{c}
E_{o} \\
E_{u}
\end{array}\right]
$$

where we use bold typeset to denote block-diagonal matrix concatenations over the sequence of observer nodes (e.g. $\left.\boldsymbol{A}_{\boldsymbol{o}}:=\operatorname{blkdiag}\left(A_{o}^{1}, \ldots, A_{o}^{N}\right)\right)$.

Due to the block-triangular structure, the errors of the observable part $E_{o}$ and unobservable part $E_{u}$ of the whole network can be treated separately such that each internal dynamics is asymptotically stable. It is evident that the block-diagonal matrix $\boldsymbol{A}_{\boldsymbol{o}}-\boldsymbol{L}_{\boldsymbol{o}} \boldsymbol{C}_{\boldsymbol{o}}$ can be made stable with an appropriate choice of the observer gains $L^{i}$ since each pair $\left(A_{o}^{i}, C_{o}^{i}\right)$ is observable. For the internal dynamics of the unobservable subspace of the error network, we can use the fact that under strong connectivity of the communication graph and joint observability the matrix $-\boldsymbol{T}_{\boldsymbol{u}}^{\top}(\mathcal{L} \otimes I) \boldsymbol{T}_{\boldsymbol{u}}$ is Hurwitz:

Proposition 1. If system (1) is jointly observable and the communication graph is strongly connected, then there exist a positive definite diagonal matrix $P$ such that

$-\boldsymbol{T}_{\boldsymbol{u}}^{\top}\left(\mathcal{L}^{\top} \otimes I\right) \boldsymbol{T}_{\boldsymbol{u}} P-P \boldsymbol{T}_{\boldsymbol{u}}^{\top}(\mathcal{L} \otimes I) \boldsymbol{T}_{\boldsymbol{u}} \prec 0$. 
This result can be inferred from the proof of Proposition 1 in 13 . For completeness, we provide a proof in the Appendix using a slightly different formulation. It follows that there exist sufficiently high consensus gains $\alpha_{1}, \ldots, \alpha_{N}>0$ such that the matrix $\boldsymbol{A}_{\boldsymbol{u}}-\boldsymbol{\alpha} \boldsymbol{T}_{\boldsymbol{u}}^{\top}(\mathcal{L} \otimes I) \boldsymbol{T}_{\boldsymbol{u}}$ is stable. Moreover, by investigating the eigenvalues of the system, the gains can be chosen such that the observer estimates converge arbitrarily fast to the state of the system.

The convergence will be exponential, that means we need an infinite amount of time to actually reach the state. Therefore, in the next section we will present a nonlinear design to obtain finite-time convergence. To prove it, we exploit the fact that the Lyapunov matrix in Proposition 1 is diagonal (i.e. $-\boldsymbol{T}_{\boldsymbol{u}}^{\boldsymbol{\top}}(\mathcal{L} \otimes I) \boldsymbol{T}_{\boldsymbol{u}}$ is diagonally stable).

\section{Finite-time distributed observer design}

In the previous section it was shown how implementing observer nodes, which employ output feedback together with consensus coupling, leads to a distributed observer design, where any exponential convergence rate can be imposed by the choice of the gain parameters. The only conditions are that the system is jointly observable and that the communication graph is strongly connected. The local observers (4) and (5) are linear, which makes the convergence only asymptotic. In this section, we present our main result, namely a design procedure for a nonlinear distributed observer such that the state estimates of the nodes converge in finite-time under the same conditions.

As in the previous section, we use the observability decomposition (2) and design a local observer for each part. The local observers for the observable part can be designed independently and in practice, any centralized method of finite-time estimation could be applied. For completeness, we present in the next subsection a well-known design based on homogeneity concepts [14. This finite-time observer structure relies on the local output to be observable, which is only the case for the observable subspace. The same approach for the whole state is not directly viable, since for none of the observer nodes the pair $\left(A, C^{i}\right)$ is observable. Instead, we exploit the fact that the consensus term in (5) introduces a term with a diagonally stable matrix in the error system (6) of the network. We show how embedding the consensus in a proper power leads to finite-time stability, again on the grounds of homogeneity arguments.

\subsection{Observable subspace}

We first consider the case where each measurement output to an observer node is scalar, as this gives rise to simple conditions for the observer design. Because each pair $\left(A_{o}^{i}, C_{o}^{i}\right)$ is observable, the observable subsystem is up to an output feedback equivalent to an integrator chain, and we can therefore directly apply the idea from [2 for the observable part of the state for every node. Using the coordinate transformation $\Theta^{i}$ into the observer canonical form
$\Theta^{i}{ }^{-1} A_{o}^{i} \Theta^{i}=\mathcal{I}-p^{i} C_{o}^{i} \Theta^{i}$, where $\mathcal{I}$ denotes an integrator chain of corresponding dimension of the observable subspace $n_{i}, p^{i}$ is the coefficient vector of the characteristic polynomial of $A_{o}^{i}$ and $C_{o}^{i} \Theta^{i}=\left[\begin{array}{lll}1 & \cdots & 0\end{array}\right]$, the observers for the observable subspace of each node can be expressed as

$\dot{\tilde{x}}_{o}^{i}=\tilde{A}_{o}^{i} \tilde{x}_{o}^{i}-\tilde{p}^{i} y_{i}-\tilde{L}_{o}^{i}\left\lceil\mathbf{1}\left(C_{o}^{i} \tilde{x}^{i}-y_{i}\right)\right\rfloor^{\gamma^{i}}$

where $\tilde{A}_{o}^{i}=\Theta^{i} \mathcal{I} \Theta^{i-1}, \tilde{p}^{i}=\Theta^{i} p^{i}$ and $\tilde{L}_{o}^{i}=\Theta^{i} \operatorname{diag}\left(L_{o}^{i}\right)$ with the observer gain $L_{o}^{i}$. For the entries of $\gamma^{i}=$ $\left[\begin{array}{lll}1+\nu_{i} & \cdots & 1+n_{i} \nu_{i},\end{array}\right]^{\top}$ we select some $-1 / n_{i}<\nu_{i}<0$ as shown in [3].

Proposition 2. Consider a system (1) and a local observer (7) for the locally observable substate $x_{o}^{i} \in \mathbb{R}^{n_{i}}$ of the system from the output $y_{i} \in \mathbb{R}$. If the entries of $L_{o}^{i}$ are selected as the coefficients of a monic Hurwitz polynomial, such that there exists matrices $P \succeq I$ and $Q \succ 0$ which satisfy the Lyapunov equation

$$
\left(\mathcal{I}-L_{o}^{i}\left[\begin{array}{lll}
1 & \cdots & 0
\end{array}\right]\right)^{\top} P+P\left(\mathcal{I}-L_{o}^{i}\left[\begin{array}{lll}
1 & \cdots & 0
\end{array}\right]\right)=-Q,
$$

and

$$
-\frac{\eta}{n_{i}\left(\sqrt{n_{i}}+\eta\right)}<\nu_{i}<0 \text { where } \eta=\frac{\lambda_{\min }(Q)}{\lambda_{\max }(P)} \frac{\exp (1)}{2\left\|P \operatorname{diag}\left(L_{o}^{i}\right)\right\|},
$$

then the estimate of the observer node will reach the locally observable substate of the system in finite-time.

In the general case, when the measurement output to an observer node is a vector $y^{i} \in \mathbb{R}^{m_{i}}$, the method from 15 for designing finite-time observers for multi-output systems can be used instead. The design procedure is more involved than in the scalar case and is thus omitted here.

\subsection{Unobservable subspace}

Based on the linear consensus coupling (5), which ensures stability with arbitrary fast but asymptotic rates, we add the same consensus term embedded in a fractional power

$$
\begin{aligned}
\dot{\tilde{x}}_{u}^{i}= & A_{r}^{i} \tilde{x}_{o}^{i}+A_{u}^{i} \tilde{x}_{u}^{i}-\alpha_{i} \sum_{j \in \mathcal{N}_{i}}\left(\tilde{x}_{u}^{i}-T_{u}^{i^{\top}} \tilde{x}_{j}\right) \\
& -\left[\sum_{j \in \mathcal{N}_{i}}\left(\tilde{x}_{u}^{i}-T_{u}^{i^{\top}} \tilde{x}_{j}\right)\right]^{\mathbf{1} \beta},
\end{aligned}
$$

where $0<\beta<1$. The intuitive idea is that just as the linear term drives the nodes into consensus and thus to the correct estimate (if each one estimates its observable part correctly), the increasing gain of the nonlinear term ensures that this happens in a finite time. We show the efficacy of this approach by analyzing the resulting error system of the unobservable part

$$
\begin{aligned}
\dot{E}_{u}= & \left(\boldsymbol{A}_{\boldsymbol{u}}-\boldsymbol{\alpha} \boldsymbol{T}_{\boldsymbol{u}}^{\boldsymbol{\top}}(\mathcal{L} \otimes I) \boldsymbol{T}_{\boldsymbol{u}}\right) E_{u}+\left(\boldsymbol{A}_{\boldsymbol{r}}+\boldsymbol{\alpha} \boldsymbol{T}_{\boldsymbol{u}}^{\boldsymbol{\top}}(\mathcal{A} \otimes I) \boldsymbol{T}_{\boldsymbol{o}}\right) E_{o} \\
& -\left\lceil\boldsymbol{T}_{\boldsymbol{u}}^{\boldsymbol{\top}}(\mathcal{L} \otimes I) \boldsymbol{T}_{\boldsymbol{u}} E_{u}-\boldsymbol{T}_{\boldsymbol{u}}^{\top} \mathcal{A} \boldsymbol{T}_{\boldsymbol{o}} E_{o}\right\rfloor^{\mathbf{1} \beta}
\end{aligned}
$$


$-\alpha(\|x\|)+\sigma(\|d\|)$ holds for all $x$ and $d$ with some $\alpha \in \mathcal{K}_{\infty}$ and $\sigma \in \mathcal{K}[20$. We use this for the following Lyapunov-

which compared to (6) has an additional nonlinear term.

The right-hand side of (9) for the case when $E_{o}=0$ (all the nodes have estimated their observable subspace correctly) can be written as a sum of homogeneous vectorfields of different degrees $\left.\dot{E}_{u}\right|_{E_{o}=0}=g_{1}\left(E_{u}\right)+g_{2}\left(E_{u}\right)$ where

$g_{1}\left(E_{u}\right)=\left(\boldsymbol{A}_{\boldsymbol{u}}-\boldsymbol{\alpha} \boldsymbol{T}_{\boldsymbol{u}}^{\top}(\mathcal{L} \otimes I) \boldsymbol{T}_{\boldsymbol{u}}\right) E_{u}$,

$g_{2}\left(E_{u}\right)=-\left\lceil\boldsymbol{T}_{\boldsymbol{u}}^{\boldsymbol{\top}}(\mathcal{L} \otimes I) \boldsymbol{T}_{\boldsymbol{u}} E_{u}\right\rfloor^{\mathbf{1} \beta}$.

Here a vector-field $f$ is called homogeneous with degree $\nu$, if for all $\lambda>0$ it holds that $f(\lambda x)=\lambda^{\nu+1} f(x)$ for all $x$. Using this definition, the linear part $g_{1}\left(E_{u}\right)$ has degree 0 and $g_{2}\left(E_{u}\right)$ has degree $\beta-1$. We can now exploit the fact that local stability at the origin can be inferred from the vector field with the lowest degree [16] (as in Lyapunov's indirect method). Furthermore, systems with homogeneous right-hand side of a certain degree have the nice property that local asymptotic stability at the origin implies global asymptotic stability or global finite-time stability at the origin depending on whether the degree is zero (e.g. the system is linear) or negative, respectively [17.

With the choice of $0<\beta<1$, we have that $g_{2}\left(E_{u}\right)$ has lower degree than $g_{1}\left(E_{u}\right)$, thus it dominates at the origin. Moreover, the degree is negative, which means that $\dot{E}_{u}=g_{2}\left(E_{u}\right)$ is finite-time stable if it is asymptotically stable at the origin. We can conclude that in this case, if (9) is globally asymptotically stable, it is also globally finite-time stable. A detailed proof of this assertion is derived in [18, Corollary 2.24] using the notion of homogeneous approximation (for a sum of homogeneous vector-fields the one with the lowest degree $\nu$ is the homogeneous approximation at the origin, since $\left.\lim _{\lambda \rightarrow 0} \lambda^{-\nu-1}\left(g_{1}(\lambda x)+g_{2}(\lambda x)\right)=g_{2}(x)\right)$.

So far we looked at the case where the error of the observable subspace $E_{o}$ of the network has attained zero. This will happen eventually in a finite time, if the observers for this part have been designed according to Section 3.1. However, until then it has to be ensured that the non-vanishing $E_{o}$ does not destabilize (9). For this, we can consider $E_{o}$ as an external input (since its dynamics is independent of $E_{u}$ ) and show that (9) is input-to-stable [19, which will also directly imply its global asymptotic stability for $E_{o}=0$.

Definition 1. A system $\dot{x}=f(x, d)$ is said to be inputto-state stable (ISS) if there exist a class $\mathcal{K} \mathcal{L}$ function $\beta$ and a class $\mathcal{K}$ function $\gamma$ such that for any initial state $x_{o} \in \mathbb{R}^{n}$ and any $d \in \mathcal{L}_{\infty}^{m}$, the solution exists for all $t \geq 0$ and satisfies

$$
\|x(t)\| \leq \beta\left(\left\|x_{0}\right\|, t\right)+\gamma\left(\sup _{s \in[0, t]}\|d(s)\|\right) .
$$

A sufficient condition for a system to be ISS is that there exists a positive definite and radially unbounded ISS Lyapunov function $V: \mathbb{R}^{n} \rightarrow \mathbb{R}_{+}$for which $\dot{V} \leq$ function borrowed from 21, which we need to prove our main result afterwards.

Lemma 1. Consider the system

$\dot{x}=M_{1} x+M_{2} f(x)+d$.

where $f(x)=\left[\begin{array}{lll}f_{1}\left(x_{1}\right) & \cdots & f_{n}\left(x_{n}\right)\end{array}\right]^{\top} \in \mathbb{R}^{n}$ is a coordinate-wise function satisfying the quadrant condition $x_{i} f_{i}\left(x_{i}\right) \geq 0, \lim _{t \rightarrow \pm \infty} f_{i}(t)= \pm \infty$ and $d \in \mathcal{L}_{\infty}^{n}$. If there exist $P \succ 0, \Lambda=\operatorname{diag}\left(\lambda_{1}, \ldots, \lambda_{n}\right) \succ 0$ and $\Upsilon=\operatorname{diag}\left(v_{1}, \ldots, v_{n}\right) \succ 0$ such that

$\Phi=\left[\begin{array}{cc}M_{1}^{\top} P+P M_{1} & M_{1}^{\top} \Lambda+P M_{2}+\Upsilon \\ * & M_{2}^{\top} \Lambda+\Lambda M_{2}\end{array}\right] \preccurlyeq 0$,

then the system (10) is ISS.

Proof. Consider a Lyapunov function $V=V_{1}+V_{2}$, where $V_{1}(x)=x^{\top} P x$ and

$V_{2}(x)=2 \sum_{i=1}^{n} \lambda_{i} \int_{0}^{x_{i}} f_{i}(s) \mathrm{d} s$,

which is positive definite and radially unbounded due to the conditions of the lemma. Taking the derivative

$\dot{V}_{1}=x^{\top}\left(M_{1}^{\top} P+P M_{1}\right) x+2 f(x)^{\top} M_{2}^{\top} P x+2 d^{\top} P x$,

$\dot{V}_{2}=f(x)^{\top}\left(M_{2}^{\top} \Lambda+\Lambda M_{2}\right) f(x)+2 x^{\top} M_{1}^{\top} \Lambda f(x)+2 d^{\top} \Lambda f(x)$

and adding and subtracting $2 x^{\top} \Upsilon f(x)+d^{\top} \Gamma d$ with some positive definite matrix $\Gamma$ leads to

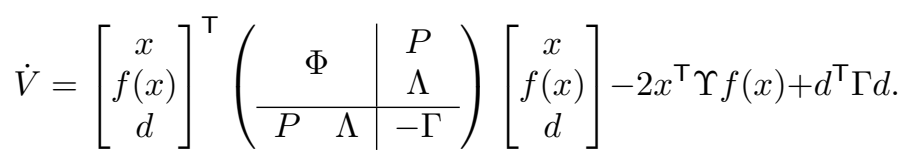

If $\Phi \preccurlyeq 0$, then there exists a $\Gamma \succ 0$ such that $\dot{V} \leq$ $-2 x^{\top} \Upsilon f(x)+d^{\top} \Gamma d$, where the first term after the inequality is negative definite and radially unbounded due to the imposed restrictions of the lemma, which implies that the system is ISS.

Uniting all facts and discussion given in this section, we are in the position to formulate our main result:

Theorem 1. Consider a system (1) which is jointly observable and a distributed observer consisting of (7) for the locally observable substate and (8) for the locally unobservable substate. Let the observers for the locally observable substates be designed according to Proposition 2 and $0<\beta<1$. If the communication graph is strongly connected, then there exist sufficiently large consensus gains $\alpha_{1}, \ldots, \alpha_{N}>0$ such that the estimates of the observer nodes will reach the state of the system in finite-time. 
Proof. The local observers for the observable substate (7) converge in finite-time under the imposed conditions of Proposition 2. It remains to show that the error dynamics of the network for the locally unobservable subspace $(90$ is ISS and that its homogeneous approximation at the origin for $E_{o}=0$ is finite-time stable. To this end, we use the affine coordinate transformation

$x=\boldsymbol{T}_{\boldsymbol{u}}^{\top}(\mathcal{L} \otimes I) \boldsymbol{T}_{\boldsymbol{u}} E_{u}-\boldsymbol{T}_{\boldsymbol{u}}^{\top} \mathcal{A} \boldsymbol{T}_{\boldsymbol{o}} E_{o}$

(the regularity of the transformation is established with Proposition 1 to arrive to at system of the form 110 where

$M_{1}=\boldsymbol{T}_{\boldsymbol{u}}^{\top}(\mathcal{L} \otimes I) \boldsymbol{T}_{\boldsymbol{u}} \boldsymbol{A}_{\boldsymbol{u}}\left(\boldsymbol{T}_{\boldsymbol{u}}^{\top}(\mathcal{L} \otimes I) \boldsymbol{T}_{\boldsymbol{u}}\right)^{-1}-\boldsymbol{T}_{\boldsymbol{u}}^{\top}(\mathcal{L} \otimes I) \boldsymbol{T}_{\boldsymbol{u}} \boldsymbol{\alpha}$, $M_{2}=-\boldsymbol{T}_{\boldsymbol{u}}^{\top}(\mathcal{L} \otimes I) \boldsymbol{T}_{\boldsymbol{u}}$

and

$$
\begin{aligned}
d= & \boldsymbol{T}_{\boldsymbol{u}}^{\top}(\mathcal{L} \otimes I) \boldsymbol{T}_{\boldsymbol{u}}\left(\boldsymbol{A}_{\boldsymbol{u}}-\boldsymbol{\alpha} \boldsymbol{T}_{\boldsymbol{u}}^{\top}(\mathcal{L} \otimes I) \boldsymbol{T}_{\boldsymbol{u}}\right) \boldsymbol{T}_{\boldsymbol{u}}^{\top} \mathcal{A} \boldsymbol{T}_{\boldsymbol{o}} E_{o} \\
& +\boldsymbol{T}_{\boldsymbol{u}}^{\top}(\mathcal{L} \otimes I) \boldsymbol{T}_{\boldsymbol{u}}\left(\boldsymbol{A}_{\boldsymbol{r}}+\boldsymbol{\alpha} \boldsymbol{T}_{\boldsymbol{u}}^{\top} \mathcal{A} \boldsymbol{T}_{\boldsymbol{o}}\right) E_{o}-\boldsymbol{T}_{\boldsymbol{u}}^{\top} \mathcal{A} \boldsymbol{T}_{\boldsymbol{o}} \dot{E}_{o}
\end{aligned}
$$

is composed by all terms which are dependent only on the estimation error $E_{o}$ of the observable part. The nonlinear functions are all $f_{i}(t)=\operatorname{sgn}(t)|t|^{\beta}$ and therefore fulfill the conditions of Lemma 1 for $\beta>0$.

Due to Proposition 1 there exist $Q \succ 0$ and a diagonal matrix $\Lambda \succ 0$ such that $M_{2}^{\top} \Lambda+\Lambda M_{2}=-Q$. It follows that $V_{2}$ is a Lyapunov-function of the homogeneous approximation $\dot{x}=-M_{2}\lceil x\rfloor^{\mathbf{1} \beta}$ with degree $\beta-1$ and is therefore finite-time stable for $0<\beta<1$.

To prove ISS of the approximated system we show that for sufficiently high $\alpha_{1}=\cdots=\alpha_{N}=\alpha>0$ the LMI (11) is fulfilled. We take $P=\alpha \Lambda$ and note that $M_{1}=S+\alpha M_{2}$, where $S=\boldsymbol{T}_{\boldsymbol{u}}^{\top}(\mathcal{L} \otimes I) \boldsymbol{T}_{\boldsymbol{u}} \boldsymbol{A}_{\boldsymbol{u}}\left(\boldsymbol{T}_{\boldsymbol{u}}^{\top}(\mathcal{L} \otimes I) \boldsymbol{T}_{\boldsymbol{u}}\right)^{-1}$. The LMI is equivalent to

$\left[\begin{array}{cc}\frac{\sqrt{\alpha}}{\alpha} I & 0 \\ 0 & \sqrt{\alpha} I\end{array}\right] \Phi\left[\begin{array}{cc}\frac{\sqrt{\alpha}}{\alpha} I & 0 \\ 0 & \sqrt{\alpha} I\end{array}\right] \preccurlyeq 0$

and after some rearrangement

$$
\left[\begin{array}{cc}
S+S^{\top} & S^{\top} \\
S & 0
\end{array}\right]-\left[\begin{array}{cc}
\Upsilon & 0 \\
0 & \Upsilon
\end{array}\right]-\left[\begin{array}{cc}
1 & 1 \\
1 & 1
\end{array}\right] \otimes(\alpha Q-\Upsilon) \preccurlyeq 0,
$$

can be shown to be fulfilled if $\left[\begin{array}{ll}\Upsilon & 0 \\ 0 & \Upsilon\end{array}\right] \succeq\left[\begin{array}{cc}S+S^{\top} & S^{\top} \\ S & 0\end{array}\right]$ and $\alpha Q \succcurlyeq \Upsilon$. Such a diagonal matrix $\Upsilon$ always exists, if $\alpha$ can be arbitrary large.

The proof of Theorem 1 guarantees the feasibility of the LMI under the same conditions of joint observability and strong connectedness as for the linear design of Section 2 . To select the consensus gains $\alpha_{i}$ it suffices to verify that 11] is fulfilled by selecting them large enough.

Remark 1. The design can be also applied in case of general directed communication graphs which are not necessarily strongly connected, by looking at the strongly connected components, identifying components which have no incoming edges as sources and requiring that the system is jointly observable only for every individual source components as in [1]. In 22] it is shown that the diagonal stability property also holds under these conditions, therefore the conditions of Theorem 1 can be relaxed to this generalized case as well.

Remark 2. Despite the fact that the analysis of this paper was given for the measurement noise-free case, the proposed observers (7) and (8) are robust with respect to measurement and state perturbations. This claim can be substantiated using the homogeneity of (7) (see [23]) and the proven ISS property of $(8)$ in Theorem 1. Homogeneous dynamical systems demonstrate also robustness against delays 24, which is an important advantage in the context of distributed estimation, where the appearance of lag is unavoidable due to the communications.

\section{Example}

Set the system's dynamics matrix as

$$
A=\left[\begin{array}{cccccc}
-1 & 0 & 0 & 0 & 0 & 0 \\
-1 & 1 & 1 & 0 & 0 & 0 \\
1 & -2 & -1 & -1 & 1 & 1 \\
0 & 0 & 0 & 1 & 0 & 0 \\
-8 & 1 & 1 & -1 & -2 & 0 \\
4 & -0.5 & 0.5 & 0 & 0 & -4
\end{array}\right]
$$

with output matrices

$$
\begin{aligned}
C_{1} & =\left[\begin{array}{llllll}
1 & 0 & 0 & 2 & 0 & 0
\end{array}\right], \\
C_{2} & =\left[\begin{array}{llllll}
2 & 0 & 0 & 1 & 0 & 0
\end{array}\right], \\
C_{3} & =\left[\begin{array}{llllll}
0 & 0 & 1 & 0 & 0 & 0
\end{array}\right], \\
C_{4} & =\left[\begin{array}{llllll}
2 & 0 & 5 & 0 & 0 & 0
\end{array}\right] .
\end{aligned}
$$

The observer network is given as in Fig. 1 .

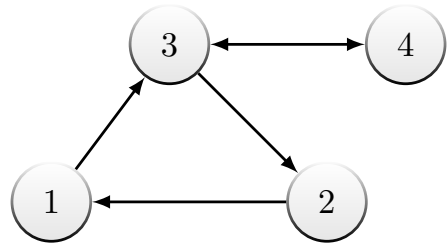

Fig. 1. Communication graph of the distributed observer.

The observer gains for the observable subspace were selected such that the eigenvalues of the error systems are all -3 for $\nu_{i}=0$ and then $\nu_{i}=-0.1$ was chosen. The consensus gains were selected to be $\alpha_{i}=10$ and $\beta=0.7$. To check if the $\alpha_{i}$ are high enough any standard LMI solver can be used to check the feasibility of (11). Fig. 2 shows the simulation result in semi-logarithmic scaling to highlight the finite-time convergence.

The result of a second simulation is shown in linear scaling in Fig. 3. where band-limited white noise of power $10^{-2}$ 


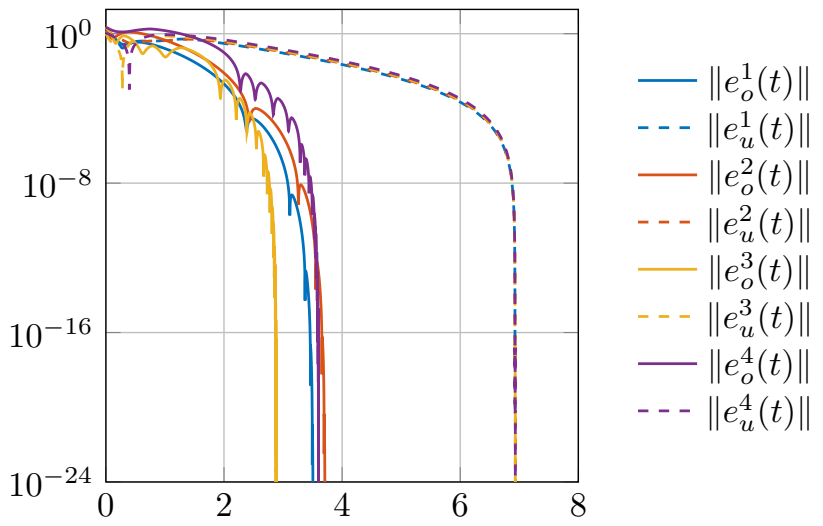

Fig. 2. Errors of the observer network in transformed coordinates.

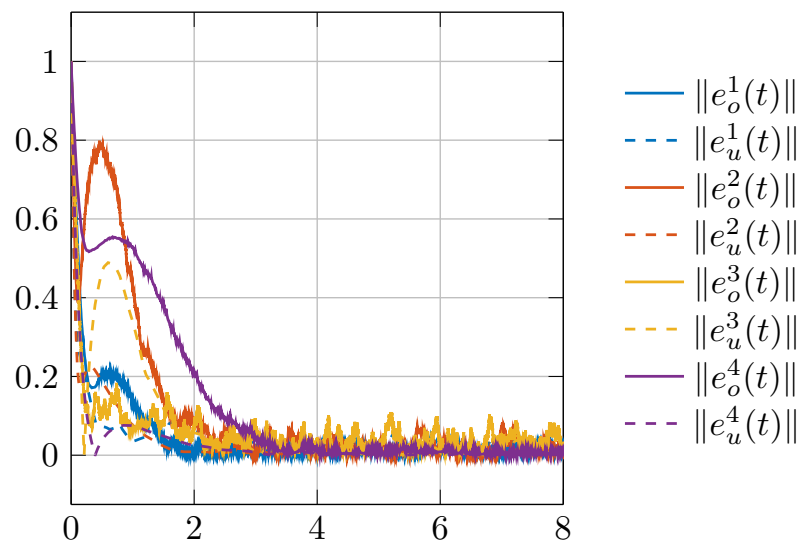

Fig. 3. Errors of the observer network in transformed coordinates in case of measurment noise.

was added to each output. It demonstrates the robustness of the distributed finite-time observer with respect to measurement noise. Note that the effect of measurement noise is more smooth in the unobservable part since it is filtered through the dynamics of the observable part of the network.

\section{Conclusion}

A finite-time distributed observer was derived using homogeneity arguments. It required that the resulting nonlinear error system for the unobservable part of the observer network is globally asymptotically stable and ISS with respect to the error in the observable part. To this end, a Lyapunov function was proposed which relied on the diagonal stability property established before. It lead to an LMI, that proved to be feasible for sufficiently large consensus gains. The design itself is simple, amounting to a common centralized design for the observable part and selecting consensus gains for the unobservable part which are verified using an LMI. Note that the finite-time observer design presented here for the distributed setting can also be used as a simple design for centralized finitetime observers for multi-output systems, by treating each entry of the output vector as it would belong to an imaginary node of a distributed observer (the connectivity and observability conditions are obviously satisfied).

The design of distributed observers has attracted increasing interest only recently (stemming for instance from the continuous integration of wireless sensor networks) and more rigorous analysis of their potential is still open. The technological applications that have been currently proposed are interesting, but a general framework of distributed observers is still missing. Nonetheless, a general motivation for distributed observers can be sought in the overall goal of having local algorithms achieving a global task. The expected benefits of decentralization, scalability and flexibility [25, 26] are left to be addressed in future works.

\section{Appendix. Proof of Proposition 1}

Proof. We first show that if system (1) is jointly observable and the communication graph is undirected and connected, then the matrix $\boldsymbol{T}_{\boldsymbol{u}}^{\boldsymbol{\top}}(\mathcal{L} \otimes I) \boldsymbol{T}_{\boldsymbol{u}}$ is positive definite.

Clearly $\boldsymbol{T}_{\boldsymbol{u}}^{\top}(\mathcal{L} \otimes I) \boldsymbol{T}_{\boldsymbol{u}} \succeq 0$, since for a connected graph $\mathcal{L}$ has a unique zero eigenvalue with corresponding eigenvector $\mathbf{1} \in \mathbb{R}^{N}$ and all the other eigenvalues are strictly larger. Assume that there exists $y^{\top}=\left[\begin{array}{lll}y^{1^{\top}} & \ldots & y^{N^{\top}}\end{array}\right] \neq 0$, $y^{i} \in \mathbb{R}^{n}$, with $y^{\top} \boldsymbol{T}_{\boldsymbol{u}}^{\top}(\mathcal{L} \otimes I) \boldsymbol{T}_{\boldsymbol{u}} y=0$, then $\boldsymbol{T}_{\boldsymbol{u}} y$ must be in $\operatorname{ker}(\mathcal{L} \otimes I)=\left\{\mathbf{1} \otimes v \mid v \in \mathbb{R}^{n}\right\}$. Due to the joint observability property $\cap_{i=1}^{N} \operatorname{Im}\left(T_{u}^{i}\right)=\{0\}$ there does not exist $v \neq 0$ and $y^{i}$ for which $T_{u}^{i} y^{i}=v$ for all $i \in\{1, \ldots, N\}$. Therefore $\boldsymbol{T}_{\boldsymbol{u}} y \notin \operatorname{ker}(\mathcal{L} \otimes I)$ for $y \neq 0$, which implies $\boldsymbol{T}_{\boldsymbol{u}}^{\boldsymbol{\top}}(\mathcal{L} \otimes I) \boldsymbol{T}_{\boldsymbol{u}} \succ 0$.

From [27] we know that if $\mathcal{L}$ is the Laplacian matrix of a strongly connected directed graph, then there exists a null vector $r$ of $\mathcal{L}^{\top}$ with strictly positive elements such that $\hat{\mathcal{L}}=\mathcal{L}^{\top} \operatorname{diag}(r)+\operatorname{diag}(r) \mathcal{L}$ is the Laplacian matrix of a connected undirected balanced weighted graph. Therefore $\boldsymbol{T}_{\boldsymbol{u}}^{\top}(\hat{\mathcal{L}} \otimes I) \boldsymbol{T}_{\boldsymbol{u}} \succ 0$, or

$\boldsymbol{T}_{\boldsymbol{u}}^{\boldsymbol{\top}}\left(\mathcal{L}^{\boldsymbol{\top}} \otimes I\right)(\operatorname{diag}(r) \otimes I) \boldsymbol{T}_{\boldsymbol{u}}+\boldsymbol{T}_{\boldsymbol{u}}^{\boldsymbol{\top}}(\operatorname{diag}(r) \otimes I)(\mathcal{L} \otimes I) \boldsymbol{T}_{\boldsymbol{u}} \succ 0$.

From $(\operatorname{diag}(r) \otimes I) \boldsymbol{T}_{\boldsymbol{u}}=\boldsymbol{T}_{\boldsymbol{u}} \operatorname{blkdiag}\left(r_{1} I_{n-n_{1}}, \ldots, r_{N} I_{n-n_{N}}\right)$ with $n-n_{i}$ being the dimension of the unobservable subspace of node $i$, we can conclude that $\operatorname{blkdiag}\left(r_{1} I_{n-n_{1}}, \ldots, r_{N} I_{n-n_{N}}\right)$ is a Lyapunov matrix for $-\boldsymbol{T}_{\boldsymbol{u}}^{\top}(\mathcal{L} \otimes I) \boldsymbol{T}_{\boldsymbol{u}}$.

\section{References}

[1] S. Park, N. C. Martins, Design of distributed LTI observers for state omniscience, IEEE Transactions on Automatic Control 62 (2) (2017) 561-576. doi:10.1109/TAC.2016.2560766

[2] S. P. Bhat, D. S. Bernstein, Geometric homogeneity with applications to finite-time stability, Mathematics of Control, Signals and Systems 17 (2) (2005) 101-127. doi:10.1007/ s00498-005-0151-x

[3] H. Silm, R. Ushirobira, D. Efimov, J.-P. Richard, W. Michiels, A note on distributed finite-time observers, IEEE Transactions on Automatic Control 64 (2) (2019) 759-766. doi:10.1109/ TAC. 2018.2838042 
[4] R. Olfati-Saber, Distributed Kalman filtering for sensor networks, in: 2007 46th IEEE Conference on Decision and Control, 2007, pp. 5492-5498. doi:10.1109/CDC.2007.4434303.

[5] V. Ugrinovskii, Distributed robust filtering with $\mathrm{H} \infty$ consensus of estimates, Automatica 47 (1) (2011) 1-13. doi:10.1016/j. automatica.2010.10.002

[6] T. Kim, H. Shim, D. D. Cho, Distributed Luenberger observer design, in: 2016 IEEE 55th Conference on Decision and Control (CDC),, 2016, pp. 6928-6933. doi:10.1109/CDC.2016.7799336

[7] W. Han, H. L. Trentelman, Z. Wang, Y. Shen, A simple approach to distributed observer design for linear systems, IEEE Transactions on Automatic Control 64 (1) (2018) 329-336. doi:10.1109/TAC.2018.2828103.

[8] Á. R. del Nozal, P. Millán, L. Orihuela, A. Seuret, L. Zaccarian, Distributed estimation based on multi-hop subspace decomposition, Automatica 99 (2019) 213-220. doi:10.1016/j. automatica.2018.10.034

[9] A. Mitra, J. A. Richards, S. Bagchi, S. Sundaram, Finite-time distributed state estimation over time-varying graphs: Exploiting the age-of-information, in: 2019 American Control Conference (ACC), 2019, pp. 4006-4011. doi:10.23919/ACC.2019. 8814627

[10] K. Zimenko, A. Polyakov, D. Efimov, Homogeneous observer design for linear MIMO systems, in: 21st IFAC World Congress, (accepted), 2020.

[11] H. Silm, R. Ushirobira, D. Efimov, W. Michiels, J.-P. Richard, E. Fridman, Comparison of the time-delay margin of a distributed and centralized observer, in: 2018 European Control Conference (ECC), Limassol, Cyprus, 2018, pp. 1963-1968. doi:10.23919/ECC.2018.8550324

[12] H. Kwakernaak, R. Sivan, Linear Optimal Control Systems, Wiley Interscience, 1972.

[13] L. Wang, J. Liu, A. S. Morse, A distributed observer for a continuous-time linear system, in: 2019 American Control Conference (ACC), 2019, pp. 86-91. doi:10.1109/TAC. 2017. 2768668

[14] W. Perruquetti, T. Floquet, E. Moulay, Finite-time observers: Application to secure communication, IEEE Transactions on Automatic Control 53 (1) (2008) 356-360. doi:10.1109/TAC. 2007.914264

[15] F. Lopez-Ramirez, A. Polyakov, D. Efimov, W. Perruquetti, Finite-time and fixed-time observer design: Implicit Lyapunov function approach, Automatica 87 (2018) 52-60. doi:10.1016/ j.automatica.2017.09.007

[16] H. Hermes, Homogeneous coordinates and continuous asymptotically stabilizing feedback controls, in: S. N. Elaydi (Ed.), Differential Equations. Stability and Control, Lecture Notes in Pure and Applied Mathematics, CRC Press, 1990, pp. 249-260.

[17] H. Nakamura, Y. Yamashita, H. Nishitani, Smooth Lyapunov functions for homogeneous differential inclusions, in: Proceedings of the 41st SICE Annual Conference. SICE 2002., Vol. 3, 2002, pp. 1974-1979 vol.3. doi:10.1109/SICE.2002.1196633.

[18] V. Andrieu, L. Praly, A. Astolfi, Homogeneous approximation, recursive observer design, and output feedback, SIAM Journal on Control and Optimization 47 (4) (2008) 1814-1850. doi: 10.1137/060675861

[19] H. K. Khalil, Nonlinear Systems, Prentice Hall, 2002.

[20] S. N. Dashkovskiy, D. V. Efimov, E. D. Sontag, Input to state stability and allied system properties, Automation and Remote Control 72 (8) (2011) 1579. doi:10.1134/S0005117911080017.

[21] D. Efimov, A. Y. Aleksandrov, Robust stability analysis and implementation of Persidskii system, in: 2019 IEEE 58th Conference on Decision and Control (CDC), 2019, pp. 6164-6168.

[22] T. Kim, C. Lee, H. Shim, Completely decentralized design of distributed observer for linear systems, IEEE Transactions on Automatic Control (in press) (2019). doi:10.1109/TAC.2019. 2962360

[23] E. Bernuau, A. Polyakov, D. Efimov, W. Perruquetti, Verification of ISS, iISS and IOSS properties applying weighted homogeneity, Systems \& Control Letters 62 (12) (2013) 1159-1167. doi:10.1016/j.sysconle.2013.09.004
[24] K. Zimenko, D. Efimov, A. Polyakov, W. Perruquetti, A note on delay robustness for homogeneous systems with negative degree, Automatica 79 (2017) 178-184. doi:10.1016/j.automatica. 2017.01 .036

[25] D. Estrin, R. Govindan, J. S. Heidemann, S. Kumar, Next Century Challenges: Scalable Coordination in Sensor Networks, in: MobiCom, 1999. doi:10.1145/313451.313556

[26] C.-Y. Chong, S. P. Kumar, Sensor networks: Evolution, opportunities, and challenges, Proceedings of the IEEE 91 (8) (2003) 1247-1256. doi:10.1109/JPROC.2003.814918

[27] W. Yu, G. Chen, M. Cao, J. Kurths, Second-order consensus for multiagent systems with directed topologies and nonlinear dynamics, IEEE Transactions on Systems, Man, and Cybernetics, Part B (Cybernetics) 40 (3) (2010) 881-891. doi: 10.1109/TSMCB.2009.2031624 